\title{
Robotic liver resection for hepatocellular carcinoma: a focus on anatomic resection
}

\author{
Yutaro Kato, Atsushi Sugioka, Ichiro Uyama \\ Department of Surgery, Fujita Health University, Toyoake, Aichi 470-1192, Japan. \\ Correspondence to: Dr. Yutaro Kato, Department of Surgery, Fujita Health University, 1-98, Dengakugakubo, Kutsukake-cho, \\ Toyoake, Aichi 470-1192, Japan. E-mail: y-kato@fujita-hu.ac.jp \\ How to cite this article: Kato Y, Sugioka A, Uyama I. Robotic liver resection for hepatocellular carcinoma: a focus on anatomic \\ resection. Hepatoma Res 2021;7:10. http://dx.doi.org/10.20517/2394-5079.2020.129
}

Received: 19 Oct 2020 First Decision: 19 Nov 2020 Revised: 30 Nov 2020 Accepted: 7 Dec 2020 Published: 7 Jan 2021

Academic Editor: Ho-Seong Han Copy Editor: Miao Zhang Production Editor: Jing Yu

\begin{abstract}
Aim: Robotic liver resection (RLR) is a new platform for minimally invasive hepatobiliary surgery. Minimally invasive surgery can confer benefits to patients with hepatocellular carcinoma (HCC), which is mostly associated with underlying chronic liver disease. Despite the inherent functional merits of robotics for surgical techniques, the clinical advantages of hepatectomy are not well defined. Therefore, we reviewed the short-term and longterm surgical results of $57 \mathrm{HCC}$ cases in 46 patients who underwent RLR at our institution.
\end{abstract}

Methods: We evaluated the feasibility and safety of robotic anatomic liver resection for HCC by comparing the results of the anatomic resection (AR) group $(n=23)$ and non-anatomic resection (NAR) group $(n=34)$.

Results: Overall $(n=57)$, the liver-specific console time was $487 \mathrm{~min}$, blood loss was $194 \mathrm{~g}$, and there was one open conversion (2\%). Postoperative data showed acceptable hepatic functional recovery, with a major complication rate of $11 \%$ and no 90 -day mortality. Compared to NAR, AR was associated with longer operative and console times, more blood loss, and worse postoperative liver function, thus reflecting the greater extent and complexity of hepatectomies for more advanced-stage tumors than NAR. Nonetheless, major complication rate, mortality rate, length of hospital stay, and RO resection rate were comparable between groups. Long-term results were comparable to those of previously reported hepatectomies for HCC and were similar between groups.

Conclusion: RLR including AR may be a safe and feasible form of hepatectomy for select patients with HCC.

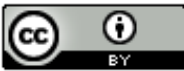

(C) The Author(s) 2021. Open Access This article is licensed under a Creative Commons Attribution 4.0 International License (https://creativecommons.org/licenses/by/4.0/), which permits unrestricted use, sharing, adaptation, distribution and reproduction in any medium or format, for any purpose, even commercially, as long as you give appropriate credit to the original author(s) and the source, provide a link to the Creative Commons license, and indicate if changes were made.

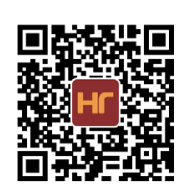


Keywords: Anatomic liver resection, Glissonian pedicle approach, hepatocellular carcinoma, laparoscopic liver resection, Laennec's capsule, robotic liver resection

\section{INTRODUCTION}

Robotic technology has increasingly infiltrated the field of abdominal surgery, encompassing urological, gynecological, and upper and lower gastrointestinal procedures ${ }^{[1,2]}$. However, the use of robotic systems is still limited in hepatobiliary and pancreatic surgery, probably because of the technical difficulty and insufficient instruments appropriate for this field. In particular, robotic liver resection (RLR) is still being developed. Several authors have reported the surgical results of RLR, and most studies have described the short-term results and compared them with those of conventional laparoscopic or open liver resections ${ }^{[3-6]}$. However, the feasibility, safety, and efficacy of RLR as a form of hepatobiliary surgery have not been well defined.

Anatomic resection (AR) of the liver is a type of hepatectomy that results in accurate and complete resection of an anatomic liver portion supplied by the corresponding portal vein branches. Therefore, AR may offer high curability, particularly for hepatocellular carcinoma (HCC), which is characterized by portal vein tumor invasion, even with small tumors ${ }^{[7]}$. Several studies have suggested the oncologic advantages of AR over non-anatomic liver resection (NAR) for patients with $\mathrm{HCC}^{[8,9]}$. Therefore, AR is a recommended form of resection for HCC in select patients, depending on their hepatic functional reserve. Although recent advances in laparoscopic liver resection (LLR) have clearly established the feasibility and safety of laparoscopic AR, it has been mostly performed by experts ${ }^{[10,11]}$. Furthermore, the feasibility and safety of robotic AR remain unestablished ${ }^{[12,13]}$.

In this single-center study of RLR for HCC, we retrospectively reviewed the short-term and long-term surgical outcomes of robotic AR and NAR performed in 57 cases in 46 patients. We describe these outcomes here along with the technical details of our standardized methods of robotic AR.

\section{METHODS}

\section{Definitions of anatomic liver resection and non-anatomic liver resection}

We defined AR as a hepatectomy procedure during which an anatomic portion of the liver parenchyma supplied by any of the first-order to fourth-order division portal vein branches is completely resected without any remaining ischemic or congestive areas. Therefore, AR was defined to include segmentectomy (I to VIII), sectionectomy (left lateral, medial, anterior, and posterior), hemihepatectomy, trisectionectomy, and any type of extended procedure to completely resect the combined anatomic segments. Subsegmentectomy, a type of resection of the specific liver areas supplied by the fourth-order division branches of the segmental Glissonian pedicles, was also included as AR. Any liver resection procedures other than AR were defined as NAR.

\section{Robotic and laparoscopic liver resections at our institute}

At our institution, we started our program of conventional LLR in May 2005 and that of RLR in December 2009. Between May 2005 and January 2020, we performed 492 minimally invasive liver resections (398 LLR and 94 RLR). One hundred sixty-one of the 398 LLR (41\%) and 46 of the 94 RLR (49\%) performed were AR. The 94 RLR were performed for 82 patients; 57 of the 94 RLR (61\%) were performed for a total of 46 patients with HCC.

\section{Patient selection}

At our institution, minimally invasive liver resection, LLR or RLR, was the first choice of approach for hepatic tumors that were $10 \mathrm{~cm}$ or smaller in size and for those that did not involve major vascular or 
Table 1. Background patient and tumor characteristics

\begin{tabular}{|c|c|c|c|c|}
\hline & All Cases $(n=57)$ & AR $(n=23)$ & NAR $(n=34)$ & $P$-value \\
\hline Age, years & $71(20-82)$ & $72(20-82)$ & $71(54-82)$ & 0.56 \\
\hline Sex, $M / F$ & $42 / 15$ & $19 / 4$ & $23 / 11$ & 0.20 \\
\hline \multicolumn{5}{|l|}{ Liver function } \\
\hline $\mathrm{TB}, \mathrm{mg} / \mathrm{dL}$ & $0.9(0.3-1.7)$ & $0.8(0.8-1.3)$ & $0.9(0.3-1.7)$ & 0.54 \\
\hline Alb, g/dL & $4.0(3.1-5.2)$ & $4.0(3.6-5.2)$ & $3.9(3.1-5.0)$ & 0.77 \\
\hline $\mathrm{AST}, \mathrm{IU} / \mathrm{L}$ & $29(14-139)$ & $25(15-69)$ & $35(14-139)$ & 0.16 \\
\hline$A L T, I U / L$ & $25(9-158)$ & $27(10-82)$ & $25(9-158)$ & 0.82 \\
\hline PT, \% & $92(28-134)$ & $92(28-127)$ & $93(60-134)$ & 0.92 \\
\hline $\mathrm{PC}, \times 10^{4} / \mathrm{mm}^{3}$ & $13.3(5.2-23.5)$ & $14.4(6.9-23.5)$ & $12.1(5.2-23.1)$ & $0.03^{\#}$ \\
\hline ICGR15, \% & $14.4(2.6-82.4)$ & $13.1(3.6-22.6)$ & $19.1(2.6-82.4)$ & $0.02^{\#}$ \\
\hline Liver cirrhosis (n) & $54 \%(31)$ & $35 \%(8)$ & $68 \%(23)$ & $0.01^{\#}$ \\
\hline \multicolumn{5}{|l|}{ Tumor characteristics } \\
\hline Tumor size, cm & $2.1(0.6-12.5)$ & $2.5(0.7-12.5)$ & $1.8(0.6-10.0)$ & $0.002^{\#}$ \\
\hline Tumor number & $1(1-6)$ & $1(1-6)$ & $1(1-5)$ & 0.53 \\
\hline Difficult segments $(n)$ & $53 \%(31)$ & $65 \%(15)$ & $47 \%(16)$ & 0.17 \\
\hline $\operatorname{AFP}(n g / m L)$ & $7.3(2.0-5811.0)$ & $4.8(2.0-5811.0)$ & $8.2(2.0-2041.0)$ & 0.33 \\
\hline $\mathrm{DCP}(\mathrm{mAU} / \mathrm{mL})$ & $26(10-95560)$ & $35(11-30899)$ & $23(10-95560)$ & 0.11 \\
\hline Recurrent tumors ( $n$ ) & $37 \%(21)$ & $30 \%(7)$ & $25 \%(14)$ & 0.41 \\
\hline \multicolumn{5}{|l|}{ UICC Stage } \\
\hline $\mid A, I B$ & 32 & 10 & 22 & \\
\hline II & 19 & 9 & 10 & $0.03^{\#}$ \\
\hline$\||| A\| \mid B$, & 4 & 4 & 0 & \\
\hline IVA, IVB & 2 & 0 & 2 & \\
\hline
\end{tabular}

AR: anatomic resection; NAR: non-anatomic resection; HCC: hepatocellular carcinoma; TB: serum total bilirubin level; Alb: serum albumin level; AST: serum asparatate aminotransferase level; ALT: serum alanine aminotransferase level; PT: prothrombin time; PC: serum platelet count; ICGR 15: indocyanine green retention rate at 15 min; Difficult segments: segments I, VII or VIII; AFP: alpha-feto protein; DCP: des-gamma-carboxy prothrombin; UICC Stage: Tumor stages based on the Union for International Cancer Control TNM classification; ${ }^{\#} P<0.05$

biliary structures needing to be reconstructed. We selected open surgery for centrally located tumors of $10 \mathrm{~cm}$ or larger with suspected invasion of the major hepatic veins. Between LLR and RLR, we do not have selection criteria. However, cases needing the extirpation of portal vein tumor thrombi were assigned to open or robotic approach only. Moreover, in cases where PVTT was not limited to the ipsilateral first-order portal vein branch, we selected open surgery.

During this study period, in contrast to LLR, RLR was not covered by national insurance and was performed at patients' own expense in our institute. This economic standpoint, along with the availability of the machine in the hospital, affected the selection of the resection approach in this study.

Selection of hepatectomy procedures or hepatectomy itself with regard to hepatic functional reserve and remnant liver volume was performed in the same way in open, laparoscopic and robotic approaches. In cases where severe adhesion was anticipated because of repeat liver resections, we chose the approaches depending on the number and types of previous liver resections, location and size of the tumor, and preoperative image findings.

\section{Patient characteristics}

The characteristics of the 57 HCC cases treated with RLR are shown in Table 1. Briefly, the median patient age at the time of surgery was 71 years, and the majority of patients were male $(n=42 ; 74 \%)$. The hepatic functional reserve of all cases was Child-Pugh class A. The median serum levels of preoperative total bilirubin (TB), albumin (Alb), aspartate aminotransferase (AST), alanine aminotransferase (ALT), prothrombin time (PT), and platelet count $(\mathrm{PC})$ were $0.9 \mathrm{mg} / \mathrm{dL}, 4.0 \mathrm{~g} / \mathrm{dL}, 24 \mathrm{IU} / \mathrm{L}, 25 \mathrm{IU} / \mathrm{L}, 92 \%$, and $13.3 \times 10^{4} / \mathrm{mm}^{3}$, respectively. The median indocyanine green (ICG) retention rate at $15 \mathrm{~min}$ (ICGR15) was $14.4 \%$. Liver cirrhosis was diagnosed in 31 patients by postoperative pathologic examination (54\%). 
Table 2. Types of RLR for HCC

\begin{tabular}{ll}
\hline Types of liver resection & $\boldsymbol{n} \mathbf{5 7}$ \\
\hline Anatomic liver resection (AR) & $n=23$ \\
(Extended) right hemihepatectomy & 2 \\
(Extended) left hemihepatectomy & 1 \\
(Extended) right anterior sectionectomy & 2 \\
(Extended) right posterior sectionectomy & 3 \\
Left lateral sectionectomy & 2 \\
(Sub)segmentectomy & 13 \\
I & 1 \\
II & 1 \\
IVa & 1 \\
V & 1 \\
V+VI & 1 \\
VI & 2 \\
VIII & 6 \\
Non-anatomic liver resection (NAR) & $n=34$ \\
II & 2 \\
III & 3 \\
IV & 6 \\
V & 5 \\
VII & 5 \\
VIII & 4 \\
\hline
\end{tabular}

RLR: robotic liver resection, " ${ }^{\#}$ locations of the largest tumor per case

\section{Tumor characteristics}

Table 1 also shows the tumor characteristics of the 57 HCC cases treated with RLR. A total of 90 HCC nodules were resected, and the median number of resected tumors per case was one. Of the 90 tumors, 41 (46\%) were located at S-I, S-VII, or S-VIII, which are generally considered difficult segments in which to perform conventional LLR. Tumors were located at the segment (S)-I, S-VII, or S-VIII in 31 of the 57 cases (53\%); $65 \%$ of these cases were treated with AR and $47 \%$ were treated with NAR. The median maximal size of the resected tumors in each case was $2.1 \mathrm{~cm}$. In 21 of the 57 cases (37\%), RLR was conducted for recurrent HCC. Tumor stages based on the Union for International Cancer Control Tumor, Node, Metastasis (TNM) classification were IA or IB for 32 cases (56\%), II for 19 cases (33\%), IIIA or IIIB for 4 cases (7\%), and IV A or IV B for 2 cases (4\%).

\section{Types of liver resection}

Table 2 shows the types of liver resection performed for the 57 RLR cases. Of these, 23 cases (40\%) were treated with AR and 34 were treated with NAR. Twenty-three resections belonged to AR in 22 patients and 34 resections belonged to NAR in 26 patients. One of the 22 patients underwent AR two times. The 26 patients undergoing NAR included 21 patients who underwent NAR only one time, 4 patients undergoing NAR multiple times and 1 patient undergoing one NAR and one AR. There were 2 patients who underwent AR in addition to NAR for multiple tumors in a single operation. These 2 patients were classified into the AR group.

For 16 of the 23 cases $(70 \%)$ treated with AR, the liver resections were major ( $\geq 3$ Couinaud's liver segments) or anatomic sectionectomies/segmentectomies for tumors located in difficult segments (S-I, S-VII, or S-VIII). Of note, for two cases (one right hemihepatectomy and one posterior sectionectomy), portal vein tumor thrombi extending into the right portal vein were extirpated by portal venous resection and reconstruction. 
A

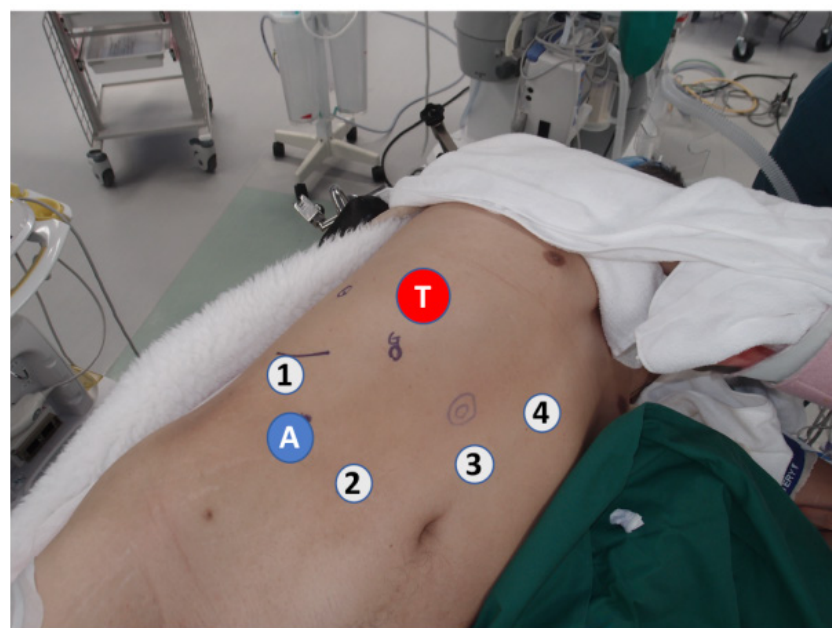

B

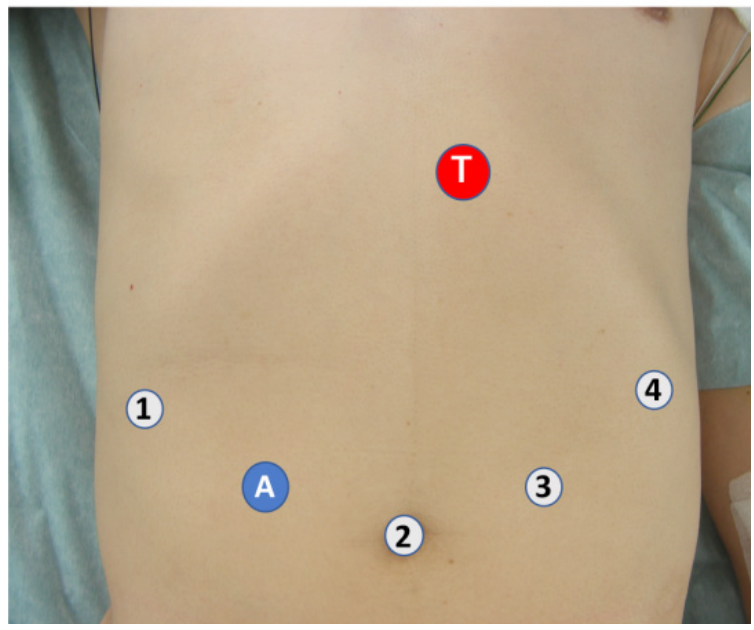

Figure 1. Patient body position and port placement. $(A)$ In the right-side resection, the patient is placed in the left decubitus or semidecubitus position with the right arm brought to the left side; (B) in the left-side resection, the patient is placed in the supine position. Robotic ports (numbers 1 to 4 ) are placed as shown. Number 2 port is usually used for endoscope. An additional 12-mm assistant trocar $(A)$ is placed between robotic ports number 1 and 2 . In both $(A)$ and (B), T represents the site of the target tumor

In 8 of the 57 cases (14\%), operative procedures other than liver resection were performed in conjunction with hepatectomy. Of these eight non-hepatectomy procedures, two were conducted along with AR (Hassab's operation with posterior sectionectomy and partial gastrectomy with segmentectomy VIII). In 21 of the 57 cases treated with RLR (37\%) (12 of the 46 patients; $26 \%$ ), repeat resections were performed using RLR. The preceding approaches were open for 8 cases, laparoscopic for 2, and robotic for 11 .

\section{Manner of analysis}

As a significant number of patients underwent multiple RLRs and two patients underwent both AR and NAR at different time points, there were discrepancies between the case number and patient number in several sets of analyses. To avoid duplication and confusion, we studied perioperative outcomes in a case-based manner $(n=57)$, and survival outcomes in a patient-based manner $(n=46)$. These manners of analyses were appropriate, not only because even if a patient underwent multiple RLRs on different dates, each session of liver resection was independent in terms of tumor and liver function characteristics, hepatectomy procedures and perioperative outcome, but also because survival analysis must be patientbased, not case-based.

\section{Surgical techniques}

All 94 RLR in our series were conducted using the da Vinci surgical system (Intuitive Surgical, Sunnyvale, CA, USA). The da Vinci S system (Intuitive Surgical) was used between 2005 and $2012(n=32)$, and the Si system (Intuitive Surgical) was used between 2013 and $2015(n=20)$. The da Vinci Xi system (Intuitive Surgical) has been used since $2017(n=42)$. The console surgeons were Y.K. and I.U., who had expertise in both LLR and RLR.

Patients were placed in the supine position for resection of left-side liver tumors and in the left semi-flank or full-flank position for resection of right-side tumors [Figure 1]. The robotic ports were placed differently for right-side and left-side resections, as shown in Figure 1. The patient cart was directed to the patient from the right cranial side. One or two laparoscopic trocars were used for surgical assistance.

Articulated instruments including Maryland bipolar forceps, curved bipolar dissector, and debakey forceps were used for most hilar dissections. Liver parenchymal resection was performed with the clamp-crush 
method using Maryland forceps or direct parenchymal division using monopolar scissors or ultrasonic shears (Harmonic ACE Curved Shears, Intuitive Surgical). Small vessels were divided using ultrasonic shears, small metal clips, or monopolar scissors, and large vessels were divided using ligature clips or linear stapler.

At our institution, there are two basic approaches to AR: the extrahepatic Glissonian pedicle approach (GPA) and hepatic vein (HV) root-at first one-way resection, regardless of the type of anatomic hepatectomy ${ }^{[14-16]}$. Using our method of AR, on the basis of the extrahepatic GPA, the target Glissonian pedicles corresponding to the anatomic liver area to be resected are isolated and occluded or divided at the hepatic hilum before parenchymal dissection is started ${ }^{[14-16]}$. This approach to the hilar Glissonian pedicles is based on the anatomical relationship between Laennec's capsule of the liver and the Glissonian sheath $^{[14-16]}$. Both structures can be manually separated from each other at the hilum by careful dissection. On the other hand, for AR requiring exposure of the major HVs, we used a technique called the HV rootat first one-way resection ${ }^{[15,16]}$. During this approach, we first dissect and expose the roots of the major HVs as a regular procedure, followed by cranial-to-caudal one-way parenchymal dissection after occlusion of the target Glissonian pedicles. This approach to the major HVs is also based on the anatomical background of Laennec's capsule ${ }^{[14]}$.

During all types of robotic AR performed in this series, we applied the extrahepatic GPA and HV rootat first one-way resection as standardized procedures; both approaches were similar to those used for conventional laparoscopic $\mathrm{AR}^{[15,16]}$. Even in anatomic segmentomies $(n=7)$ or subsegmentectomies $(n=6$; subsegment VIII-c: $n=3$, VIII-acd: $n=1$, IV-a: $n=1$, VI-a: $n=1$ ), we isolated and controlled the third- or fourth- order pedicles extrahepatically at the hilum, before starting parenchymal dissection.

We used intraoperative ultrasonography for detection of tumors, acquisition of resection margin and confirmation of vascular anatomy and flow. In the early period of this study, we used conventional laparoscopic ultrasonography, which was operated by the assistant surgeon. In the later period, a dedicated probe was used directly by the console surgeon.

The use of the Pringle maneuver was restricted to cases where bleeding during parenchymal dissection was not controlled well. When the Pringle maneuver was applied, we snared the hepatoduodenal ligament using a tape and tourniquet it with a Nelaton catheter, which was brought out through the abdominal wall along with the tape inside it.

\section{Representative procedures during robotic AR: robotic anatomic segmentectomy VIII}

Robotic AR procedures that are used in robotic anatomic segmentectomy VIII are shown in Figures 2-4. Cystic plate cholecystectomy ${ }^{[14,16]}$ is the first procedure; it allows good access to the root of the Glissonian pedicle of the anterior section (G-ant). At the hilar plate, several pieces of fibrous tissue connect the plate and liver parenchyma, which we call the anchor ${ }^{[14]}$. By dividing the anchors, one can easily create and enter the space between the liver parenchyma covered with Laennec's capsule and Glissonian pedicle sheaths ${ }^{[14]}$. Laennec's capsule-based layer dissection at Gates IV and V according to the Gate theory ${ }^{[14]}$ facilitates extrahepatic isolation of the G-ant by passing a tape from Gate IV to Gate V [Figure 2A and B]. Compared to the open procedure, laparoscopic and robotic approaches seem to be more useful for meticulous and accurate layer dissection and isolation of pedicles due to the magnified caudal view of the hilum. Furthermore, during robotic dissection of the hilar pedicles, articulated instruments are quite useful.

Next, the ventral surface of the G-ant is dissected, and the pedicle of segment V (G-V) is isolated extrahepatically. The right stump of the tape holding G-ant is then passed under G-V and switched cranially over G-V, resulting in the isolation of the G-VIII pedicle (G-VIII) extrahepatically [Figure 2C]. 

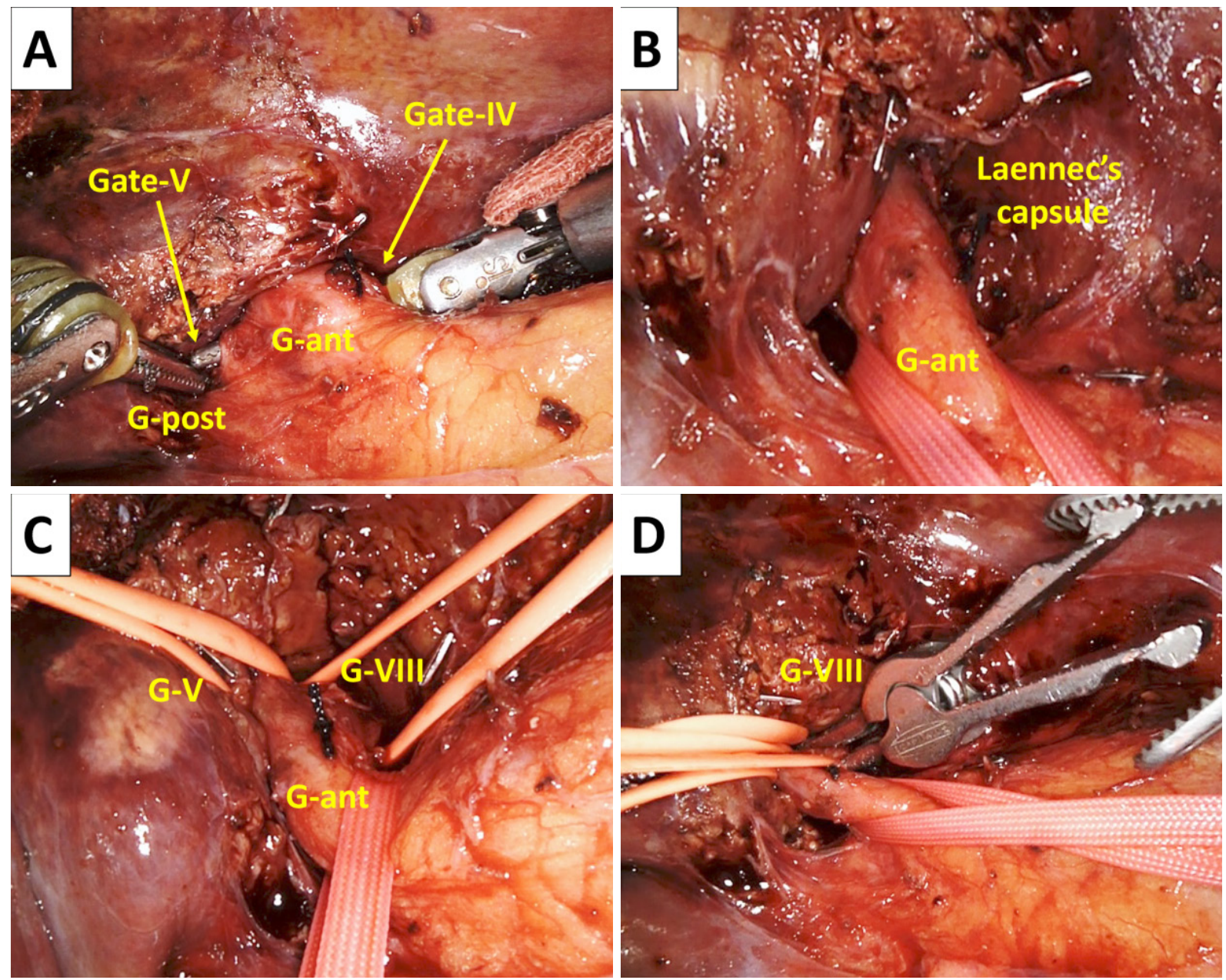

Figure 2. Hilar dissection in robotic anatomic segmentectomy VIII using the extrahepatic Glissonian pedicle approach (GPA). (A) Passing articulated forceps from the Gate IV to V to encircle the Glissonian pedicle of the right anterior section (G-ant). G-post denotes the Glissonian pedicle of the right posterior section; (B) the G-ant encircled extrahepatically. The shining Laennec's capsule of the segment IV parenchyma is shown; (C) the Glissonian pedicles of the segments V (G-V) and VIII (G-VIII) are taped extrahepatically; (D) the G-VIII is clamped using a bulldog clamp

A

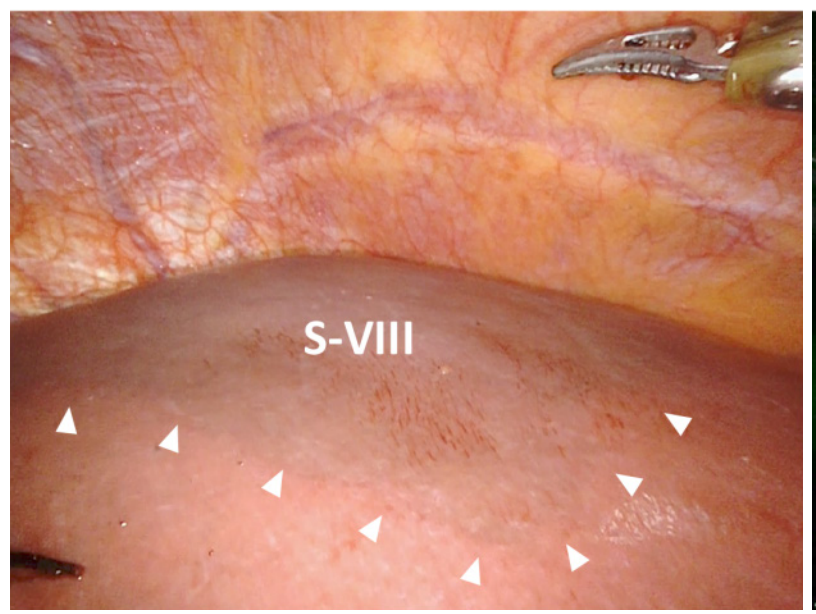

B

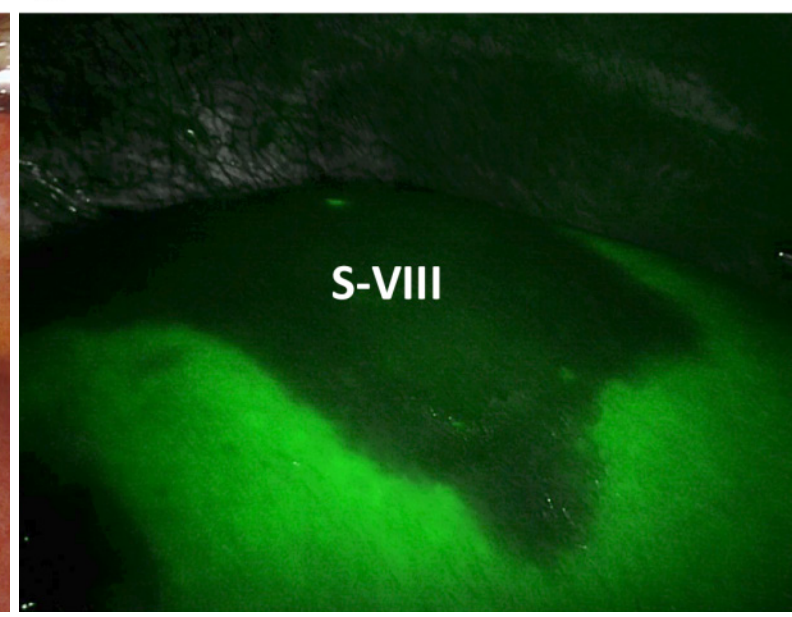

Figure 3. Discoloration of the segment VIII (S-VIII) after a specific clamping of G-VIII. (A) Normal endoscopic view. Note the ischemic $\mathrm{S}$-VIII as the discolored area (arrows); (B) firefly mode using the intravenous injection of indocyanine green (ICG). S-VIII is recognized as the negative-stained area 

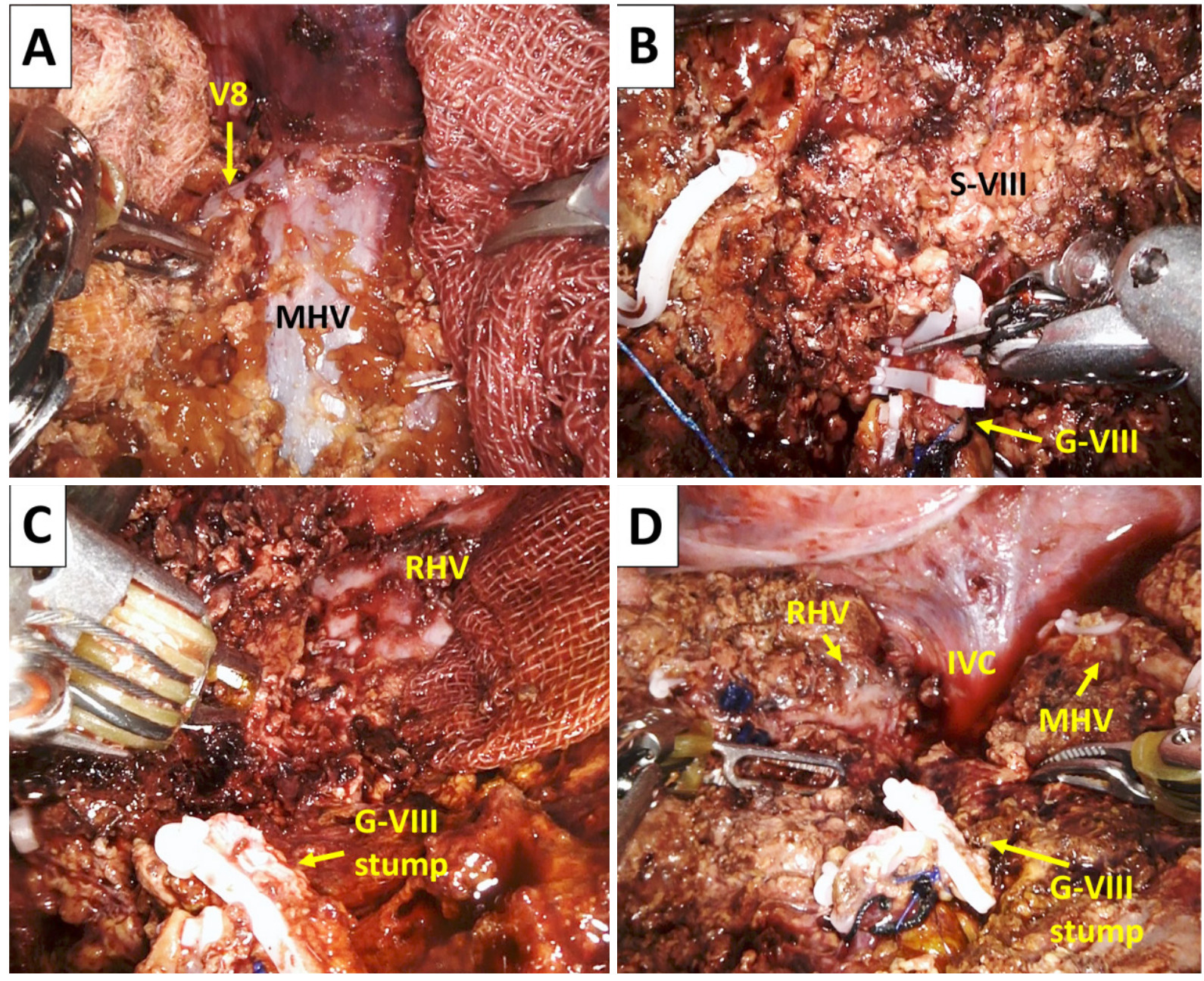

Figure 4. The HV-root at first one-way parenchymal resection during segmentectomy VIII. (A) the root and anterior wall of the middle hepatic vein (MHV) and a tributary V8 are exposed by HV-root at first parenchymal dissection using monopolar scissors (by the right hand) and bipolar fenestrated forceps (by the left hand); (B) in the middle of the cranial-to-caudal parenchymal dissection, the G-VIII, which was already controlled during hilar preparation, is divided; (C) the root of the right hepatic vein (RHV) is exposed cranially. The divided stump of the G-VIII is shown; (D) the final operative view after completion of a robotic anatomic segmentectomy VIII. The inferior vena cava (IVC) and cranial parts of the MHV and RHV are exposed. The divided stump of G-VIII is also seen

This method of isolating deeper pedicles is called the subtraction method ${ }^{[14-16]}$. By clamping G-VIII [Figure 2D], the isolated S-VIII becomes ischemic before parenchymal dissection is started [Figure 3A]. Intravenous injection of ICG after clamping G-VIII clearly confirms S-VIII as a negatively stained area in the Firefly mode [Figure 3B].

After confirmation of the discoloration of S-VIII, parenchymal dissection is started by exposing the root of the middle hepatic vein (MHV), which is tracked and exposed from the root side to the peripheral side in the cranial-to-caudal direction (i.e., HV root-at first one-way resection) [Figure 4A]. The root of the right hepatic vein (RHV) is similarly exposed, and parenchymal dissection continues along the RHV in the cranial-to-caudal direction. Several MHV tributaries from the S-VIII were divided. In the middle of parenchymal resection, G-VIII, which was already isolated at the hilum, is fully exposed on the dorsal side of the MHV. G-VIII is then divided at a level that does not jeopardize G-V and G-ant [Figure 4B]. Several G-VIII branches can be divided individually. Division of G-VIII is followed by parenchymal dissection in the left-to-right and cranial-to-caudal directions, and anatomic segmentectomy VIII is completed at the right edge of the S-VIII [Figure $4 \mathrm{C}$ and D]. We do not use the Pringle maneuver routinely during anatomic segmentectomy VIII. 


\section{Postoperative complications}

Postoperative complications were described according to the Clavien-Dindo (C-D) classification ${ }^{[17]}$. Major complications were defined as those with C-D grade $\geq$ IIIa. Complications at the surgical-site were defined as those that might be related to technical failure requiring medical, radiological, or surgical intervention, such as bile leak, fluid collection, and abscess formation on the liver resection plane. Systemic complications were defined as those that induced cardiac, pulmonary, renal, or cerebral disorders.

We encountered several RLR and LLR cases complicated by severe acute kidney injury (AKI) and intraoperative $\mathrm{CO}_{2}$ gas embolism with or without acute respiratory distress syndrome. As we suspected that the AirSeal system (ConMed, Utica, NY, USA) for pneumoperitoneum was related to these events, we abandoned this system in mid-2018. Thereafter, we created a rule stating that pneumoperitoneum must be paused for 5-10 min every 2-3 h during surgery and that lung recruitment by manual ventilation with positive end-expiratory pressure must be conducted during the paused phase.

\section{Statistical analysis}

Continuous data are expressed as median (range). Quantitative data and categorical data were compared using the Kruskal-Wallis test and Pearson's chi-square test, respectively. Cumulative overall survival (OS) and disease-free survival (DFS) after surgery were analyzed by the Kaplan-Meier method; when necessary, they were compared between groups using the log-rank test. $P<0.05$ was considered statistically significant. Statistical analyses were performed using JMP Pro software (v14.2.0; SAS Institute Inc., Cary, NC, USA).

\section{Study design}

Medical records were searched and data were collected from the charts. The study was conducted with the approval of the Institutional Regulation Board (approval number: HM19-064) and in accordance with the Declaration of Helsinki (2000). Written informed consent was obtained from all patients.

\section{RESULTS}

Table 1 shows the characteristics of all 57 cases and compares the data of the AR $(n=23)$ and NAR $(n=$ 34) groups. Patient age, sex distribution, and preoperative serum levels of TB, Alb, AST, ALT, and PT were comparable between groups. Although all cases were Child-Pugh class A, the more precisely estimated liver functional reserve, as judged by the serum PC and ICGR15, was significantly better in the AR group than in the NAR group. The rate of (histologically proven) liver cirrhosis was significantly higher in the NAR group than in the AR group ( $68 v$ s. $35 \% ; P=0.01$ ). Tumor size was significantly greater in the AR group than in the NAR group $(2.5 \mathrm{~cm} v s .1 .8 \mathrm{~cm} ; P=0.002)$, and the tumor number per case was comparable in both groups $(n=1)$. Difficult tumor locations (S-I, S-VII, or S-VIII), recurrent tumors, and serum levels of alpha-fetoprotein (AFP) and des-gamma-carboxy prothrombin were comparable between groups. These results suggest that patients who underwent AR had a significantly better hepatic functional reserve than those who underwent NAR, and that larger tumors tended to be resected by AR. Tumor stages were I or II for 51 of the 57 cases (89\%); when the AR and NAR groups were compared, tumor stages were significantly higher in the AR group $(P=0.03)$.

\section{Short-term results of RLR for HCC}

\section{Intraoperative data}

Table 2 shows the short-term outcomes of the 57 HCC cases who underwent RLR and a comparison of the AR $(n=23)$ and NAR $(n=34)$ groups. The median total operative time, including that for robot docking, laparoscopic adhesiolysis before docking, and other adjunctive non-hepatic procedures, was $612 \mathrm{~min}$, and the median liver-specific console time (LSCT), which represented the console time only for liver surgery, was $487 \mathrm{~min}$. Total operative time ( $837 \mathrm{~min} v s .445 \mathrm{~min} ; P<0.0001)$ and LSCT $(703 \mathrm{~min} v s$. 
Table 3. Short-term outcomes

\begin{tabular}{|c|c|c|c|c|}
\hline & All cases $(n=57)$ & AR $(n=23)$ & NAR $(n=34)$ & $P$-value \\
\hline \multicolumn{5}{|l|}{ Intraoperative data } \\
\hline Total operative time ${ }^{\star}, \min$ & $612(58-2154)$ & $837(328-2154)$ & $445(58-1107)$ & $<0.0001^{\#}$ \\
\hline $\mathrm{LSCT}^{\star \star}, \min$ & $487(46-1957)$ & $703(245-1957)$ & $271(46-850)$ & $<0.0001^{\#}$ \\
\hline Concurrent proc. $(n)$ & $14 \%(8)$ & $9 \%(2)$ & $18 \%(6)$ & 0.33 \\
\hline $\mathrm{EBL}, \mathrm{g}$ & $194(5-6900)$ & $336(43-6900)$ & $135(5-4876)$ & $0.009^{\#}$ \\
\hline Pringle maneuver $(n)$ & $12 \%(7)$ & $26 \%(6)$ & $3 \%(1)$ & $0.008^{\#}$ \\
\hline Open conversion $(n)$ & $2 \%(1)$ & $4 \%(1)$ & $0 \%(0)$ & 0.22 \\
\hline \multicolumn{5}{|l|}{ Postoperative laboratory data } \\
\hline Max TB, mg/dL & $1.5(0.7-5.9)$ & $1.5(1.0-5.9)$ & $1.4(0.7-3.6)$ & 0.16 \\
\hline Max AST, IU/L & $433(64-4844)$ & $877(64-4844)$ & $292(65-2106)$ & $0.001^{\#}$ \\
\hline Min PT, \% & $63(40-88)$ & $58(40-77)$ & $69(43-88)$ & $0.005^{\#}$ \\
\hline $\operatorname{Min} P C, \times 10^{4} / \mathrm{mm}^{3}$ & $7.5(3.4-17.2)$ & $8.2(3.8-17.2)$ & $7.4(3.4-16.1)$ & 0.47 \\
\hline \multicolumn{5}{|l|}{ Pathological data } \\
\hline $\mathrm{RO}(n)$ & $98 \%(56)$ & $96 \%(22)$ & $100 \%(34)$ & 0.22 \\
\hline Hospital stay, days & $15(8-82)$ & $16(8-82)$ & $15(8-34)$ & 0.22 \\
\hline \multicolumn{5}{|l|}{ Postoperative complications } \\
\hline$\geq$ C-D grllla $(n)$ & $11 \%(6)$ & $17 \%(4)$ & $6 \%(2)$ & 0.16 \\
\hline 90-day mortality $(n)$ & $0 \%(0)$ & $0 \%(0)$ & $0 \%(0)$ & 1.0 \\
\hline
\end{tabular}

AR: anatomic resection; NAR: non-anatomic resection; total operative time*: operative time including the time required for extrahepatic procedures, if applicable; LSCT**: liver-specific console time, console time only for liver procedures; EBL: estimated blood loss; Max: maximum value; Min: minimum value; TB: total bilirubin; AST: asparatate aminotransferase; PT: prothrombin time; PC:platelet count; C-D gr: Clavien-Dindo grade; ${ }^{*} P<0.05$

$271 \mathrm{~min} ; P<0.0001)$ were significantly longer in the AR group than in the NAR group. The concurrent extrahepatic procedure rates were similar between groups. The median estimated blood loss (EBL) was $194 \mathrm{~g}$; it was significantly greater in the AR group than in the NAR group (336 g vs. $135 \mathrm{~g} ; P=0.009$ ). The Pringle maneuver was applied in 7 of 57 cases (12\%); its rate was significantly higher in the AR group than in the NAR group ( 26 vs. $3 \% ; P=0.008)$. Open conversion was performed for one case treated with AR $(4 \%)$ for bleeding.

Postoperative laboratory and pathological data and hospital stay

Postoperative laboratory data showed that serum levels of AST and PT were significantly higher in the AR group than in the NAR group [Table 3], suggesting a greater resection volume for AR cases, although changes in TB and PC levels were comparable between groups. The pathological Ro rate was similarly high between groups ( 96 vs. 100\%). There was one case of R1 resection in the AR group. In this case, the tumor at the posterior section had multiple daughter nodules and PVTT extending to the right portal vein. We performed a posterior sectionectomy with PVTT extirpation. A few daughter nodules were exposed along the resection plane with a margin of zero, which was diagnosed as R1. The length of hospital stay (median, 15 days) was similar in the AR group (16 days) and NAR group (15 days) $(P=0.22)$.

\section{Postoperative complications}

The overall rate of postoperative C-D grade $\geq$ IIIa complications was $11 \%(n=6)$. Furthermore, the postoperative C-D grade $\geq$ IIIa complication rates were similar in the AR group $(n=4 ; 17 \%)$ and NAR group $(n=2 ; 6 \%)(P=0.16)$ [Table 3]. The details of the documented C-D grade $\geq$ IIIa complications $(n=7)$ are shown in Table 4. The rates of surgical-site C-D grade $\geq$ IIIa complications were $0 \%$ in the AR group and $3 \%$ in the NAR group ( $n=1$; bile leak); this difference was not statistically significant. Six of the seven $(86 \%)$ events were systemic complications [Table 4], and the rate of systemic complications was higher in the AR group than in the NAR group (17 vs. 3\%; $P=0.06)$. Of note, postoperative AKI occurred in three patients; of these, two experienced an intraoperative massive $\mathrm{CO}_{2}$ gas embolism with severe hypotension that was likely associated with the use of the AirSeal system (ConMed) for pneumoperitoneum. Neither 
Table 4. Major postoperative complications ( $\geq$ Clavien-Dindo grade IIla)

\begin{tabular}{|c|c|c|c|c|c|}
\hline C-D grade & Number of events & Details of events & All $(n=57)$ & AR $(n=23)$ & NAR $(n=34)$ \\
\hline IIla & 3 & $\begin{array}{l}\text { pleural effusion }{ }^{\star} \\
\text { bile leak }\end{array}$ & $\begin{array}{l}2 \\
1\end{array}$ & $\begin{array}{l}2 \\
0\end{array}$ & $\begin{array}{l}0 \\
1\end{array}$ \\
\hline IIIb & 0 & & 0 & 0 & 0 \\
\hline IVa & 3 & $\begin{array}{l}\mathrm{AKI}^{*} \\
\text { cerebral infarction* }\end{array}$ & $\begin{array}{l}2 \\
1\end{array}$ & $\begin{array}{l}2 \\
0\end{array}$ & $\begin{array}{l}0 \\
1\end{array}$ \\
\hline Vibe & 1 & ARDS and $A K I^{*}$ & 1 & 1 & 0 \\
\hline V & 0 & & 0 & 0 & 0 \\
\hline Total & 7 & & 7 & 5 & 2 \\
\hline
\end{tabular}

AR: anatomic resection; NAR: non-anatomic resection; C-D grade: Clavien-Dindo grade; AKI: acute kidney injury; ARDS: acute respiratory distress syndrome. *defined as systemic complications

AKI nor pulmonary complications occurred in 21 cases after we stopped using the AirSeal system (ConMed) and introduced our protocol involving intermittent pausing of pneumoperitoneum and concurrent lung recruitment. The 90 -day mortality and in-hospital mortality rates were $0 \%$ in both groups.

\section{Survival data}

The cumulative OS and DFS rates after the first RLR of 46 patients with HCC were analyzed. The OS rates were 98,82 , and $74 \%$, and the DFS rates were 80,42 , and $31 \%$ at 1,3 , and 5 years after the first RLR, respectively [Figure 5]. The median OS rate was not reached. The median DFS length was 25.9 months after surgery. For 21 HCC patients who underwent robotic AR as the first RLR, the OS rates were 95, 86, and $86 \%$, and the DFS rates were 75,37 , and $37 \%$ at 1,3 , and 5 years after the first RLR, respectively [Figure 6]. For 25 HCC patients who underwent robotic NAR as the first RLR, the OS rates were 100, 81 , and $71 \%$, and the DFS rates were 74,41 , and $29 \%$, respectively, at 1, 3, and 5 years after the first RLR, respectively [Figure 6 ]. The OS and DFS rates were not statistically different between the AR and NAR groups (OS: $P=0.54$; DFS: $P=0.55$; log-rank test).

\section{DISCUSSION}

This review evaluated the surgical results of 57 RLR cases that were composed of 46 HCC patients at a single center over the course of the past 10 years. Furthermore, we described the particular surgical techniques for robotic AR based on the extrahepatic GPA and HV root-at first one-way resection in detail. We standardized AR using these two basic techniques for open liver resection and $\operatorname{LLR}^{[15,16]}$, and we were able to apply the same techniques to the aforementioned 23 robotic AR cases. Additionally, the inherent functional merits of robotics, including the instrument articulation and stabilized operative field, may have contributed to precise hilar dissection during the extrahepatic GPA. However, insufficient instruments for parenchymal dissection remain a problem. Harmonic shears (Intuitive Surgical) are useful for parenchymal resection on a straightforward line such as that in hemihepatectomy, but they may be difficult to use or require expertise in cases of a curved or complex resection plane, as in segmentectomies VII and VIII. During such difficult AR procedures, we have to use the clamp-crush method with articulated forceps or vessel sealers, which may frequently require the Pringle maneuver. Further development of instruments suitable for parenchymal resection is required.

In our experience consisting of 94 RLRs, HCC was the indication for most procedures $(n=57 ; 61 \%)$. Although the majority of HCC cases were relatively early-stage tumors (Union for International Cancer Control stages I and II: 89\%), the patient and tumor characteristics were not favorable; there were considerable rates of underlying cirrhosis (54\%), tumors located in laparoscopically difficult segments (53\%), repeat liver resections (37\%), and concomitant extrahepatic procedures (14\%). Nonetheless, the overall $(n=57)$ postoperative short-term and long-term outcomes were considered acceptable compared to the previously reported results of RLR for HCC or other types of tumors ${ }^{[3-6,13,18-22]}$. The operative time 
A

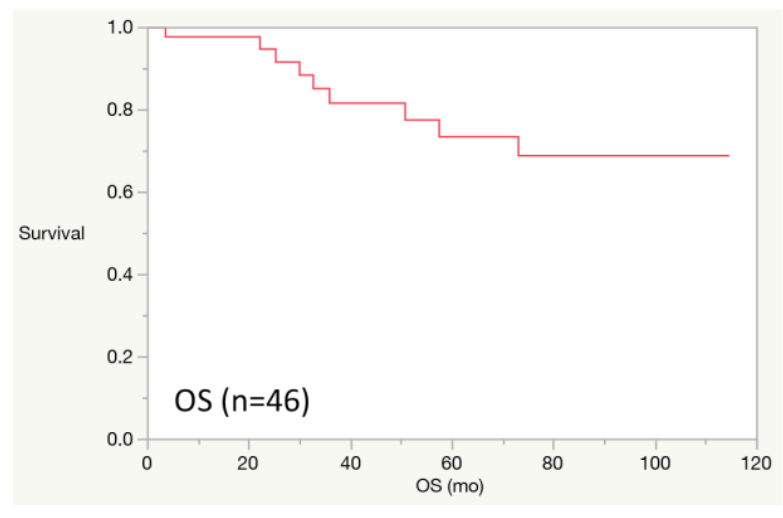

B

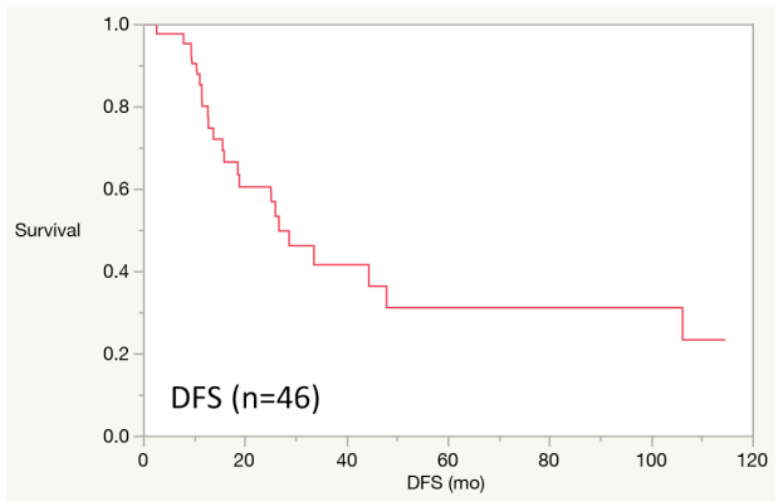

Figure 5. Overall and disease-free survivals after the first RLR in 46 patients with HCC. (A) overall survival (OS); (B) disease-free survival (DFS)

A

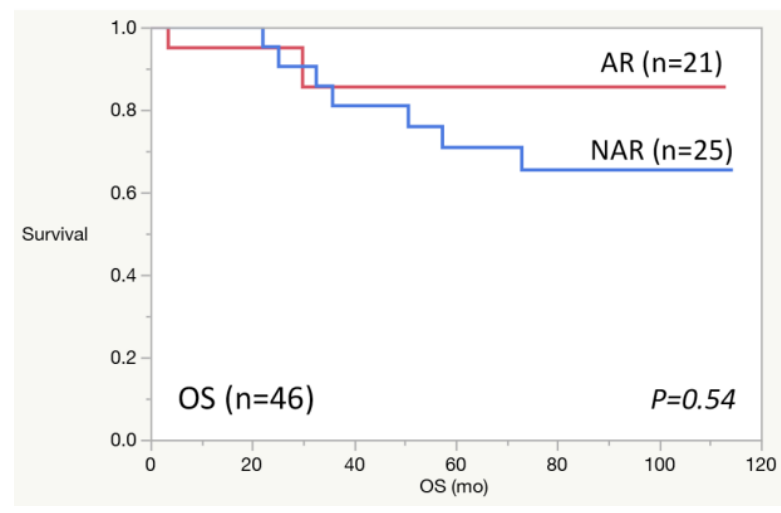

B

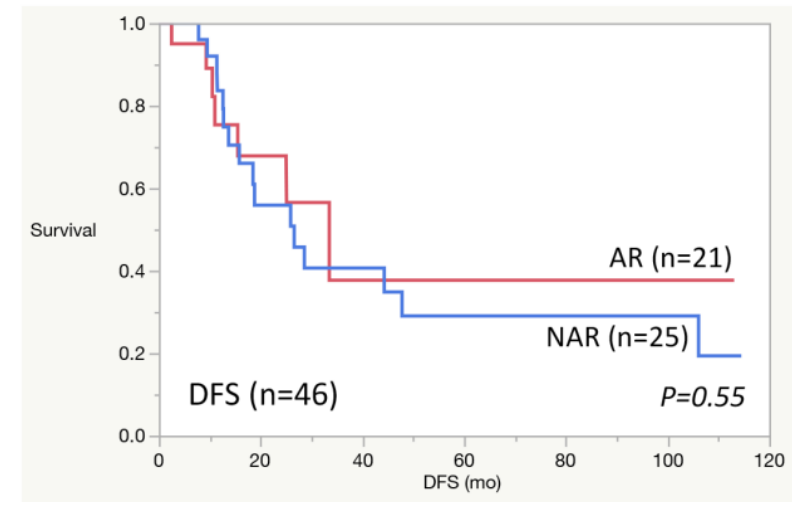

Figure 6. Comparison of survivals between the AR $(n=21)$ and NAR $(n=25)$ groups. (A) overall survival (OS); (B) disease-free survival (DFS). There were no statistical differences in OS or DFS between the two groups

and length of hospital stay for AR in our series appeared longer than that previously reported for $\mathrm{HCC}^{[20-22]}$. However, accurate comparisons are difficult due to the differences in patients, tumors, and procedures between the studies.

To further evaluate the feasibility and safety of robotic AR for HCC, we compared the outcomes of the AR and NAR, because the feasibility and safety of RLR have been established for NAR by previous studies ${ }^{[18-21]}$. Our results showed that, compared to NAR, AR was associated with longer operative times, longer lower critical solution temperature (LCST), more EBL, higher Pringle maneuver application rates, higher postoperative AST, and lower PT. These findings are reasonable in view of the greater extent and complexity of hepatectomy in the AR group. However, it is notable that although the procedures were more complex, extended, and technically demanding, and although the tumor stages were significantly higher in the AR group than in the NAR group, the major complication rates, mortality rates, lengths of hospital stay, and Ro resection rates were not statistically different between groups. Considering the better hepatic functional reserve in the $\mathrm{AR}$ group, these results suggest that despite the need for technical expertise in robotic AR, it may be a safe and feasible type of liver resection for HCC in selected patients.

Close examination of postoperative complications in 57 RLR cases revealed that six of the seven major events (86\%) were systemic complications, and most of these were considered related to the AirSeal system 
(ConMed). In these cases, grave $\mathrm{CO}_{2}$ gas embolism with significant hypotension was observed, even with minor HV injuries; this sometimes occurred without significant gas embolism during LLR or even RLR using a conventional pneumoperitoneal machine. Furthermore, no systemic complications occurred after discontinuing the use of the AirSeal system (ConMed). These findings and the mechanical actions of the AirSeal system (ConMed) collectively imply that we should avoid using this system, particularly during $\mathrm{AR}$, where the major HVs are routinely exposed during parenchymal dissection. The AirSeal systemrelated severe complications are not limited to RLR. In fact, we experienced $\mathrm{CO}_{2}$ embolism in a case of AR exposing a major HV during LLR. On the other hand, the rates of surgical-site complications were very low ( $1.8 \%$ for 57 RLR and $0 \%$ for 23 AR cases). These promising results suggest the potential advantages of robotics, such as topical technical safety, particularly for AR; however, a comparison of surgical-site complication rates among open, laparoscopic, and robotic AR using similar techniques ${ }^{[15,16]}$ is warranted.

Previous studies compared the short-term results of RLR and LLR for HCC ${ }^{[20,21]}$. They reported that operative time, blood loss, complication rate, and length of hospital stay were similar using the two approaches. The authors also suggested that RLR may offer advantages over LLR in major AR procedures or when tumors are in difficult locations, such as posterosuperior segments ${ }^{[20,21]}$. Further studies are necessary to determine the advantages and disadvantages of RLR compared to LLR for HCC. Moreover, comparative studies of robotic and laparoscopic AR for HCC are warranted to clarify the real significance of robotic AR for this disease entity.

The long-term results showed that the OS rate of our 46 HCC patients who underwent RLR seemed acceptable compared to that reported by previous studies ${ }^{[20,23]}$. Moreover, the respective OS and DFS rates of the AR and NAR groups seemed acceptable, and the outcomes of both groups were similar. Larger studies are needed to further define the oncologic effects of RLR or robotic AR on the treatment of HCC.

There are several difficulty scoring systems used in $\operatorname{LLR}^{[24,25]}$. We have not used these systems for assessing the difficulty in RLR cases. We think that these systems may be also useful for RLR, but additional factors may be considered such as the resection types requiring the curved resection plane in the absence of ultrasonic aspirator, port placement to reduce robotic arm interference and patient body position to facilitate optimal arm movement. Regarding the learning curve for RLR, we consider that the curve may be more low-gradient in RLR than in LLR, if the robotic beginner has already significant experience with LLR. However, AR is still more difficult in RLR than in LLR because of inadequate instruments for parenchymal dissection for RLR, though the hilar dissection and vascular control and hemostasis are easier in RLR than in LLR.

There have been no reported randomized control studies comparing RLR and LLR. At our institution, indication of surgery and procedures are similar between the two approaches. However, both the economic issue as a result of no insurance coverage on RLR and the issue of machine availability in the hospital preclude randomized studies. Propensity-score matched studies and future randomized studies are warranted.

There are limitations in this study. First, this is a retrospective study with a small sample size. Second, as stated above, the economic issue resulting from no insurance coverage on RLR greatly affected selection of approaches.

In conclusion, RLR may be a safe and feasible form of hepatectomy for HCC. Robotic AR for HCC can be technically standardized with acceptable safety by the GPA and HV root-at first one-way resection. However, further experience and comparative studies of RLR, LLR, and open resection are necessary to define the significance of robotic AR for the treatment of HCC. 


\section{DECLARATIONS}

\section{Acknowledgments}

The authors thank Akira Yasuda, Takeshi Takahara, Yoshinao Tanahashi, Masayuki Kojima, Sanae Nakajima, Gozo Kiguchi, Yuichiro Uchida and Junichi Yoshikawa for their substantial contributions to surgery and preparation of the manuscript.

We would like to thank Editage (www.editage.com) for English language editing.

\section{Authors' contributions}

Concepts and design of the study and data acquisition, analysis and interpretation: Kato Y, Sugioka A, Uyama I

Performance of surgery: Kato Y, Uyama I

Technical support: Sugioka A, Uyama I

Administrative and material support: Sugioka A, Uyama I

\section{Availability of data and materials}

The datasets are not available for public access due to patient privacy concerns but are available from the corresponding author on reasonable request.

\section{Financial support and sponsorship}

None.

\section{Conflict of interest}

All authors declared that there are no conflicts of interest.

\section{Ethical approval and consent to participate}

The study was conducted under the approval of Institutional Regulation Board (approval number: HM19064) and in accordance with the Declaration of Helsinki (2000). Written informed consent was obtained from all patients.

\section{Consent for publication}

Written informed consent for publication was obtained from all patients.

\section{Copyright}

(c) The Author(s) 2021.

\section{REFERENCES}

1. Suda K, Nakauchi M, Inaba K, Ishida Y, Uyama I. Robotic surgery for upper gastrointestinal cancer: current status and future perspectives. Dig Endosc 2016;28:701-13.

2. Andolfi C, Umanskiy K. Appraisal and current considerations of robotics in colon and rectal surgery. J Laparoendosc Adv Surg Tech A 2019;29:152-58

3. Ocuin LM, Tsung A. Robotic liver resection for malignancy: Current status, oncologic outcomes, comparison to laparoscopy, and future applications. J Surg Oncol 2015;112:295-301.

4. Qiu J, Chen S, Chengyou D. A systematic review of robotic-assisted liver resection and meta-analysis of robotic versus laparoscopic hepatectomy for hepatic neoplasms. Surg Endosc 2016;30:862-75.

5. Tsung A, Geller DA, Sukato DC, et al. Robotic versus laparoscopic hepatectomy: a matched comparison. Ann Surg 2014;259:549-55.

6. Kingham TP, Leung U, Kuk D, et al. Robotic liver resection: a case matched comparison. World J Surg 2016;40:1422-8.

7. Makuuchi M. Surgical treatment for HCC--special reference to anatomical resection. Int J Surg 2013;11:S47-9.

8. Shindoh J, Makuuchi M, Matsuyama Y, et al. Complete removal of the tumor-bearing portal territory decreases local tumor recurrence and improves disease-specific survival of patients with hepatocellular carcinoma. $J$ Hepatol 2016;64:594-600.

9. Kishi Y, Hasegawa K, Kaneko J, et al. Resection of segment VIII for hepatocellular carcinoma. Br J Surg 2012;99:1105-12.

10. Hasegawa Y, Nitta H, Takahara T, et al. Safely extending the indications of laparoscopic liver resection: When should we start 
laparoscopic major hepatectomy? Surg Endosc 2017;31:309-16.

11. Wakabayashi G, Cherqui D, Geller DA, et al. Recommendations for laparoscopic liver resection: a report from the second international consensus conference held in Morioka. Ann Surg 2015;261:619-29.

12. Ji WB, Wang HG, Zhao ZM, Duan WD, Lu F, Dong JH. Robotic-assisted laparoscopic anatomic hepatectomy in China: initial experience. Ann Surg 2011;253:342-8.

13. Lee JH, Han DH, Jang DS, Choi GH, Choi JS. Robotic extrahepatic Glissonean pedicle approach for anatomic liver resection in the right liver: techniques and perioperative outcomes. Surg Endosc 2016;30:3882-8.

14. Sugioka A, Kato Y, Tanahashi Y. Systematic extrahepatic Glissonean pedicle isolation for anatomical liver resection based on Laennec's capsule: proposal of a novel comprehensive surgical anatomy of the liver. J Hepatobiliary Pancreat Sci 2017;24:17-23.

15. Kato Y, Sugioka A, Tanahashi Y, et al. Standardization of Laparoscopic Left Hemihepatectomy by Extrahepatic Glissonean Pedicle Isolation and HV Root - At First One-way Resection Based on Laennec's Capsule. SGO 2020;25:78.

16. Kato Y, Sugioka A, Tanahashi Y, et al. Standardization of Anatomic Segmentectomy VIII by Extrahepatic Glissonean Pedicle Isolation and HV Root - At First One-way Resection Based on Laennec's Capsule: Open and Laparoscopic Approaches. SGO 2020;25:85.

17. Dindo D, Demartines N, Clavien PA. Classification of surgical complications: a new proposal with evaluation in a cohort of 6336 patients and results of a survey. Ann Surg 2004;240:205-13.

18. Giulianotti PC, Coratti A, Sbrana F, et al. Robotic liver surgery: results for 70 resections. Surgery 2011;149:29-39.

19. Nota CL, Rinkes IHB, Molenaar IQ, et al. Robot-assisted laparoscopic liver resection: a systematic review and pooled analysis of minor and major hepatectomies. HPB 2016;18:113-120.

20. Lai ECH, Tang CN. Long-term Survival Analysis of Robotic Versus Conventional Laparoscopic Hepatectomy for Hepatocellular Carcinoma: A Comparative Study. Surg Laparosc Endosc Percutan Tech 2016;26:162-6.

21. Magistri P, Tarantino G, Guidetti C, et al. Laparoscopic versus robotic surgery for hepatocellular carcinoma: the first 46 consecutive cases. J Surg Res 2017;217:92-9.

22. Han DH, Choi SH, Park EJ, Kang DR, Choi GH, Choi JS. Surgical outcomes after laparoscopic or robotic liver resection in hepatocellular carcinoma: a propensity-score matched analysis with conventional open liver resection. Int J Med Robot 2016;12:735-42.

23. Kudo M, Izumi N, Sakamoto M, et al. Survival analysis over 28 years of 173,378 patients with hepatocellular carcinoma in Japan. Liver Cancer 2016;5:190-7.

24. Ban D, Tanabe M, Ito H, et al. A novel difficulty scoring system for laparoscopic liver resection. $J$ Hepatobiliary Pancreat Sci 2014;21:745-53.

25. Hasegawa Y, Wakabayashi G, Nitta H, et al. A novel model for prediction of pure laparoscopic liver resection surgical difficulty. Surg Endosc 2017;31:5356-63. 